\title{
Convergence of Human Genetics and Animal Studies: Gene Therapy for X-Linked Retinoschisis
}

\author{
Ronald A. Bush ${ }^{1}$, Lisa L. Wei ${ }^{2}$, and Paul A. Sieving ${ }^{2}$ \\ ${ }^{1}$ National Institute on Deafness and Other Communication Disorders, Bethesda, Maryland 20892 \\ ${ }^{2}$ National Eye Institute, Bethesda, Maryland 20892 \\ Correspondence: bushr@nidcd.nih.gov
}

Retinoschisis is an X-linked recessive genetic disease that leads to vision loss in males. Xlinked retinoschisis (XLRS) typically affects young males; however, progressive vision loss continues throughout life. Although discovered in 1898 by Haas in two brothers, the underlying biology leading to blindness has become apparent only in the last 15 years with the advancement of human genetic analyses, generation of XLRS animal models, and the development of ocular monitoring methods such as the electroretinogram and optical coherence tomography. It is now recognized that retinoschisis results from cyst formations within the retinal layers that interrupt normal visual neurosignaling and compromise structural integrity. Mutations in the human retinoschisin gene have been correlated with disease severity of the human XLRS phenotype. Introduction of a normal human retinoschisin cDNA into retinoschisin knockout mice restores retinal structure and improves neural function, providing proof-of-concept that gene replacement therapy is a plausible treatment for XLRS.

$X_{n}$ linked retinoschisis (XLRS) is a developmental retinal genetic disease that impairs vision, typically beginning in young males and progressing into older age. Although the condition was first recognized in the 19th century and is readily diagnosed clinically by the characteristic intraretinal cysts arranged in a "spokewheel" pattern in the center of the macula, scientific progress lagged until the gene was identified about 15 years ago. This led to understanding the molecular and cellular basis of the condition, discerning the structure and function of the retinoschisin protein, generating XLRS animal models, developing suitable delivery vectors for the back of the eye, and methods to monitor structure and function of the retina in animals and humans. The culmination of these scientific discoveries poises the field for near-term clinical testing of a genetic therapy designed to restore vision and prevent further vision loss in XLRS patients.

\section{CLINICAL DISEASE}

Human retinoschisis leads to vision loss that affects boys, typically at a young age. The disease was first described in two brothers in Germany in 1898 (Haas 1898). The term retinoschisis was first used to describe the condition by Wilczek (1935) and refers to the abnormal splitting

Editors: Eric A. Pierce, Richard H. Masland, and Joan W. Miller

Additional Perspectives on Retinal Disorders: Genetic Approaches to Diagnosis and Treatment available at www.perspectivesinmedicine.org

Copyright (C) 2015 Cold Spring Harbor Laboratory Press; all rights reserved; doi: 10.1101/cshperspect.a017368

Cite this article as Cold Spring Harb Perspect Med 2015;5:a017368 
R.A. Bush et al.

through the retinal neurosensory layers. Retinoschisis is quite different from retinal detachment, in which the neurosensory tissue is elevated off and detached from the underlying retinal pigment epithelium. In 1938, the disease was recognized as an $\mathrm{X}$-linked recessive trait (Mann and MacRae 1938). Today, clinical prevalence of retinoschisis is approximately 1:5000 to $1: 25,000$ males worldwide (George et al. 1995). Vision impairment is characteristically noticed in young males before grade school, although on occasion infants may manifest severe disease. Being an X-linked macular dystrophy, the trait is passed from mothers to affected males. Female carriers nearly never display retinal abnormalities and have normal electroretinogram (ERG) responses. On very rare occasions, female carriers will show white flecks or areas of schisis within the peripheral retina (Kaplan et al. 1991). However, because the vision of female carriers is not impacted, this indicates that haploinsufficiency does not alter apparent retinal architecture nor result in obvious visual deficiencies.

Individuals with congenital XLRS manifest a broad spectrum of clinical phenotypes, thus making initial diagnosis challenging. Schisis can involve the superficial, middle, and deep retinal layers, and is seen in both the nuclear and plexiform layers. Retinoschisis may involve the peripheral retina (Prenner et al. 2006) and lead to increased incidence of full thickness retinal detachment. In general, patients with $\mathrm{X}$-linked juvenile retinoschisis show a mild reduction in central visual acuity, which gradually decreases, ranging from 20/60 to 20/120. Figure 1 shows the hallmarks of the clinical signs in the retina of a patient with a spokewheel pattern of tiny cysts that reigns in the fovea (Fig. 1A). These cavities are also readily seen by optical coherence tomography (OCT) (Fig. 1B).

The ERG is one of the most clinically informative tests for $\mathrm{X}$-linked retinoschisis. The dark-adapted b-wave in XLRS patients is reduced, whereas the a-wave amplitude often remains normal (Fig. 1C) (Peachey et al. 1987). This can result in an electronegative response in which the positive-going b-wave does not increase above baseline. As the a-wave originates from photoreceptors, this indicates relatively normal photoreceptor activity. The b-wave originates from bipolar cells that lie proximal to the photoreceptors, and the electronegative response implicates defective synaptic transmission. Although an electronegative ERG response is not exclusive to XLRS individuals, only a few

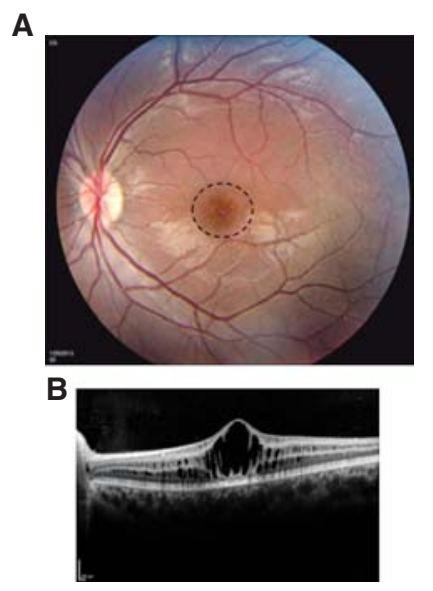

C

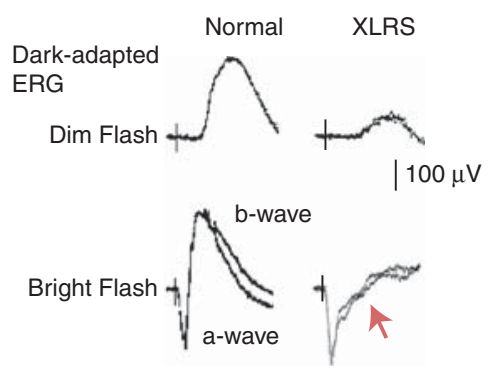

Figure 1. Fundus photo, OCT scan, and ERG typical of patients with XLRS. Color photo of macular region in X-linked retinoschisis patient $(A)$ showing the characteristic intraretinal cysts arranged in a "spokewheel" pattern and corresponding OCT scan $(B)$. Dark-adapted ERGs from both scotopic dim and bright stimulus flashes for an XLRS patient shows a characteristic reduction in b-wave amplitude relative to the preserved a-wave $(C)$. 
other retinal dystrophies result in this waveform configuration. Hence, finding this ERG type in a suspect male with a family history of retinoschisis is clinical confirmation of XLRS. However, some affected males have a more normal ERG, as was reported previously (Sieving et al. 2000; Bowles et al. 2011; Vincent et al. 2013)

\section{MANAGEMENT OF XLRS}

Currently, there are no approved treatments for the retinal degeneration or for the retinal schisis cavities. Various experimental treatment options have been considered, including the use of carbonic anhydrase inhibitors (e.g., dorzolamide and acetazolamide) with the presumed mechanism of action being the reduction of excess intraretinal fluid in cystic spaces (Apushkin and Fishman 2006; Ghajarnia and Gorin 2007). In one study, seven of eight patients showed a decrease in foveal lesions in one eye as evaluated by OCT, and six patients were reported to have a modest improvement in visual acuity (Apushkin and Fishman 2006). These findings have not been replicated and are not universally accepted. Treatment of a retinoschisin-deficient mouse model of XLRS (see below) with dorzolamide failed to produce short-term or long-term structural improvement in the retina (Zhour et al. 2012). Another proposed intervention is manipulation of the retina through surgical intraocular vitrectomy that may reduce schistic cavities (Ikeda et al. 2008). Unfortunately, these approaches do not address the fundamental pathophysiology, and no claims have been made that they alter the clinical course.

\section{GENE AND CELL BIOLOGY OF XLRS}

Genetic mutations causing XLRS are in the Retinoschisin 1 ( $R S 1)$ gene, which maps to Xp22 and is composed of six exons (Sauer et al. 1997). Retinoschisin is a 224 amino acid secretory protein that is present throughout the neural retina (Grayson et al. 2000; Molday et al. 2001; Reid et al. 2003; Takada et al. 2004). Retinoschisin gene expression has been localized to photoreceptors in both human (Grayson et al. 2000) and mouse retina (Reid et al. 1999; Grayson et al. 2000;
Molday et al. 2001; Takada et al. 2004), as well as to neurons in the inner nuclear layer (Molday et al. 2001; Takada et al. 2004), and ganglion cell layer (Takada et al. 2004) of mouse.

RS1 has a signal peptide sequence that allows for transport of the protein to the extracellular surface (Vijayasarathy et al. 2006, 2007). It also contains a single discoidin domain (Sauer et al. 1997; Grayson et al. 2000). Discoidin domains are associated with a family of extracellular cell surface proteins that are involved in cell adhesion and signaling and that interact with collagens to regulate extracellular matrix modeling (Vogel et al. 2006). Ten cysteine residues within the retinoschisin discoidin domain are important for appropriate protein folding and the formation and function of retinoschisin dimers and octamers (Wu and Molday 2003). RS1 is thought to be critical for cell adhesion during the normal development and maintenance of retinal structure. This is consistent with other discoidin domain-containing proteins and is supported by clinical observations in patients and in mouse models.

Retinoschisin expression begins during early postnatal retinal development and is maintained throughout adult life (Takada et al. 2004). As Figure 2 shows, retinoschisin is produced in the inner retina, namely, the ganglion cells, early in ocular development (top panel). However, as the eye develops, additional retinal neurons produce retinoschisin, including the outer retinal cells such as photoreceptors (bottom panel), as indicated by the presence of retinoschisin on the inner segments of rods. Interestingly, RS1 protein is also associated with bipolar cells, suggesting that it may be critical for maintaining the structural connection of postsynaptic and presynaptic elements in the outer plexiform layer that is necessary for the transmission of a visual signal from photoreceptors to bipolar cells (Molday et al. 2001; Reid et al. 2003; Takada et al. 2004). This implies that monitoring synaptic integrity by the ERG bwave would be a suitable means for gauging this connectivity. These findings support the hypothesis that retinoschisin plays a critical role in maintaining cell-cell adhesion and in preserving retinal structure and function. 
R.A. Bush et al.

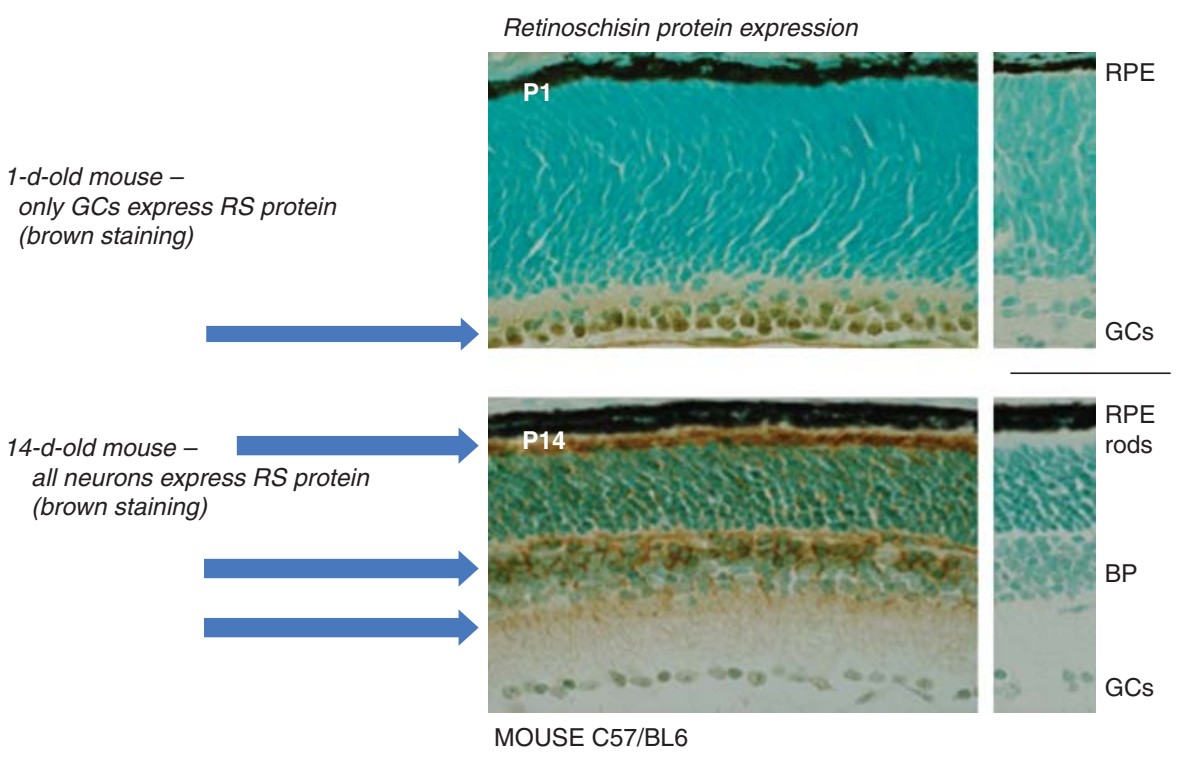

Figure 2. Retinoschisin protein expression in the mouse eye begins immediately following neuronal development and is maintained during adult life. Early in development retinoschisin is produced in the retinal ganglion cells (P1, top panel); later, extensive retinoschisin expression is also observed in the photoreceptors, including the rod inner segments (P14, bottom panel). (From Takada et al. 2004; reprinted, with express permission from the authors.)

\section{HUMAN GENETICS FOR RS1 MUTATIONS}

In XLRS patients, numerous RS1 mutations have been identified (The Retinal Consortium 1998; Eksandh et al. 2000; Simonelli et al. 2003). Mutations reported include missense changes, deletions, insertions, and splice site variants. Structure-function analyses reveal that the most common variants, missense mutations, interfere by three primary means: (1) interrupting normal secretion of retinoschisin; (2) disrupting proper octomerization; or (3) impacting normal function of the protein. Interestingly, a large proportion of these mutations are within the discoidin domain (exons 4-6), suggesting that the discoidin domain is a critical component for RS1 function. Examination of the nature of the $R S 1$ gene mutations predicts that $\sim 40 \%$ of all the identified mutations represent null alleles and, therefore, would not produce functional retinoschisin protein (Molday et al. 2012). Such mutations represent the human equivalent of genetic knockout models in mice (see below). The majority of cases found with missense mutations had minimal structure perturbations and consequently, standard disease (Sergeev et al. 2010). However, removal or insertions of cysteine residues or changes in the hydrophobic core of retinoschisin resulted in maximum structural perturbations that caused significant changes in photopic b/a ratios (Sergeev et al. 2013). Although difficult to show, in some cases, severity of disease correlates with genotype (Sergeev et al. 2010, 2013; Bowles et al. 2011).

\section{X-LINKED RETINOSCHISIS MOUSE MODELS}

Currently, three XLRS mouse models targeting the murine ortholog of the human RS1 gene have been developed (Table 1). All are deficient in endogenous murine retinoschisin. One model was engineered with the insertion of a LacZ/ $\mathrm{Neo}^{\mathrm{r}}$ cassette into exon 3, thus generating a null mouse model. Zeng et al. (2004) inserted a neomycin gene into intron 1 that resulted in ablation of murine retinoschisin protein expression. The last mouse model system was produced using $N$-ethyl-N-nitrosurea (ENU)-based ran- 
Table 1. Comparison of XLRS mouse models

\begin{tabular}{|c|c|c|c|c|}
\hline Source & Synonyms & Target & Category & $\begin{array}{c}\text { Reported } \\
\text { abnormal } \\
\text { phenotypes }\end{array}$ \\
\hline $\begin{array}{l}\text { Bernhard H.F. Weber } \\
\text { (Weber et al. 2002) }\end{array}$ & $R s 1 h^{-/ Y}$ & $\begin{array}{l}\text { Partial deletions of } \\
\text { exon } 3 \text { and } 4 \text { and } \\
\text { intron } 3 \text { with a Lac } \\
\text { Z gene insertion }\end{array}$ & $\begin{array}{l}\text { Targeted } \\
\quad \text { (reporter) }\end{array}$ & $\begin{array}{l}\text { Nervous system, } \\
\text { vision/eye }\end{array}$ \\
\hline $\begin{array}{l}\text { Paul A. Sieving } \\
\quad \text { (Zeng et al. 2004) }\end{array}$ & $R s 1^{-/ Y}$ & $\begin{array}{l}\text { Deletion of all of exon } 1 \\
\text { and partial deletion } \\
\text { of intron } 1 \text { with a } \\
\text { neomycin gene } \\
\text { insertion }\end{array}$ & $\begin{array}{l}\text { Targeted } \\
\quad \text { (knockout) }\end{array}$ & Vision/eye \\
\hline $\begin{array}{l}\text { Tennessee Mouse Genome } \\
\text { Consortium (TMGC)- } \\
\text { Monica Jablonski } \\
\text { (distributed via TMGC, } \\
\text { Northwestern University, } \\
\text { and JAX Labs) } \\
\text { (Jablonski et al. 2005) }\end{array}$ & $\begin{array}{c}\text { 44TNJ } \\
R s 1^{\mathrm{tmgcl}} / R s 1^{\mathrm{tmgcl}} \\
\text { and } R s 1^{\mathrm{tmgc}} / \mathrm{Y} \\
\text { formerly called } \\
R s 1 h^{\mathrm{tmgcl}}\end{array}$ & $\begin{array}{l}\text { T to } C \text { transition at the } \\
\text { second base of intron } \\
2 \text { of the Rsih gene }\end{array}$ & $\begin{array}{l}\text { Chemically } \\
\text { induce } \\
\text { (ENU) }\end{array}$ & $\begin{array}{l}\text { Nervous system, } \\
\text { pigmentation, } \\
\text { vision/eye }\end{array}$ \\
\hline
\end{tabular}

dom mutagenesis that led to a single base-pair substitution in intron 2 (Jablonski et al. 2005). This created an alternate splice site producing two transcripts in male mice: a 10 bp insertion and a 26 bp deletion at the junction of exon 2 to intron 2. Within these two transcripts, premature stop codons are formed, preventing synthesis of full-length retinoschisin protein. All three knockout models display structural and functional retinal phenotypes very similar to that observed in humans with XLRS. ERG evaluations in the three mouse models show the characteristic reduction of the dark-adapted bwave as observed in XLRS patients. Histologically, retina structure is disorganized, with misaligned cells and retinal splitting evident in the inner nuclear layer and structural abnormalities of synapses within the outer plexiform layerhallmarks of the human disease. Figure 3 shows the retinal structure and dampened ERGs in the $R s 1^{-/ Y}$ mouse (Zeng et al. 2004).

\section{GENE THERAPY FOR XLRS}

\section{Gene Delivery Considerations}

The expression of retinoschisin $(R s 1)$ in mice during development and in adult tissue, along with the pathology described in the XLRS mouse retina, suggests that retinoschisin is important in normal development and maintenance of the retinal architecture, and this protein appears to be critical in sustaining functional synaptic integrity (Takada et al. 2004; Kjellstrom et al. 2007). These findings support the strategy of gene augmentation as a therapeutic approach for XLRS patients. Experimental studies introducing human RS1 in mouse models of XLRS showed that a single administration gave sustained expression beyond a year and restored both structure and function of the retina (Zeng et al. 2004; Min et al. 2005). Based on these encouraging studies, efforts are now directed to develop a gene therapy approach for XLRS patients.

A first consideration involves selecting an appropriate means to deliver the gene. There are several methods that could be used to introduce the $R S 1$ gene into the eye. Viral vectors have typically shown the best efficiency with regard to delivering the gene of interest to retinal cells. Within the eye, several viral vectors have advanced or are advancing into clinical testing. Among these are vectors based on adeno-associated virus (AAV), adenovirus, retrovirus, and lentivirus (Song et al. 2003; Chevez-Barrios 
R.A. Bush et al.
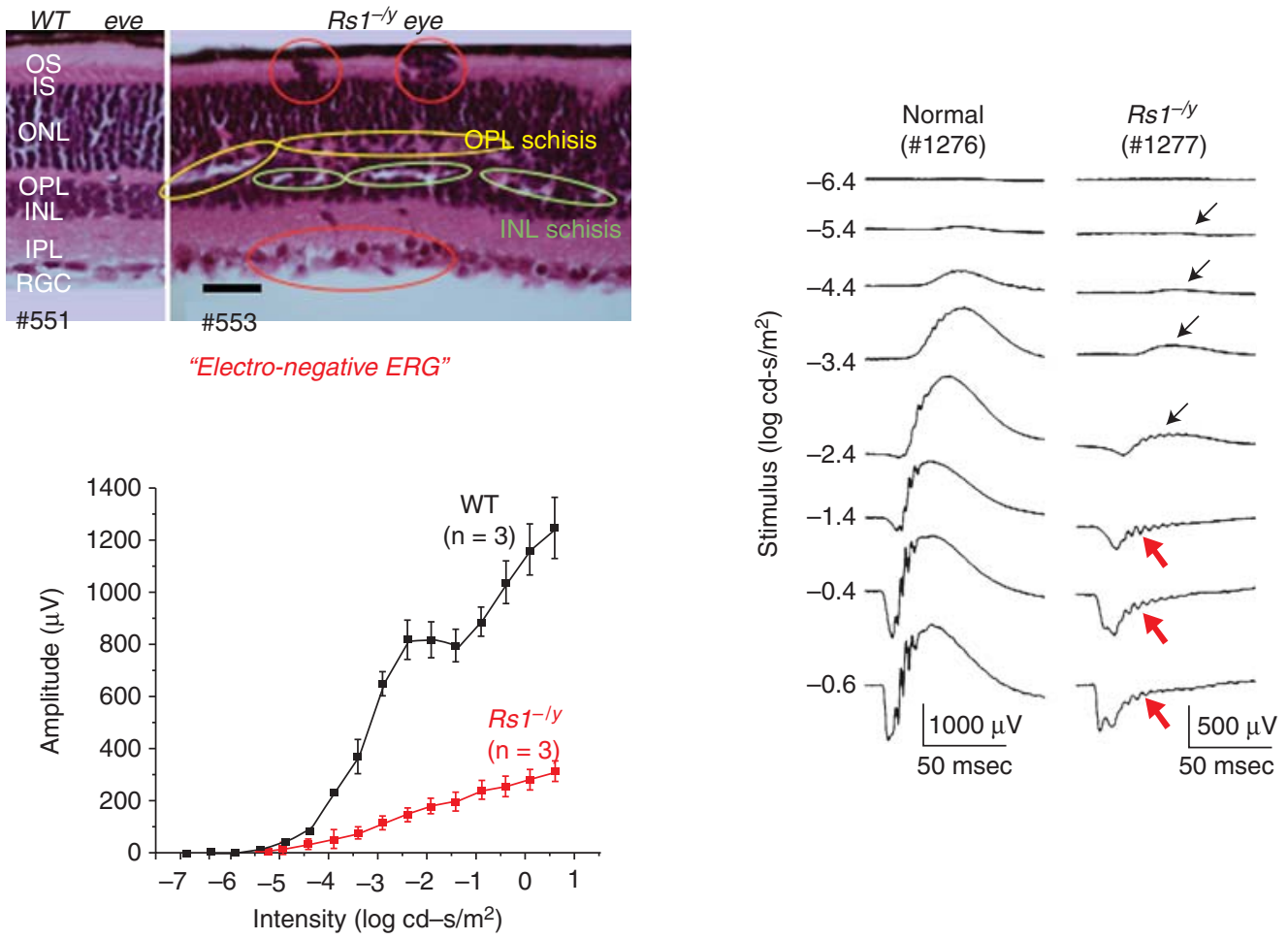

Figure 3. Retinal structure and ERG function in the Rs1-KO $\left(\mathrm{Rs}^{-/ y}\right)$ mouse. The retinal sections show structural changes between the normal mouse eye and the RS-KO mouse eye. The Rs1-KO eye shows distortions in the retinal ganglion cell layer (RGC) and random cells in the inner plexiform layer (IPL). Likewise, the synaptic connections between photoreceptors and bipolar cells are irregular in the inner nuclear layer (INL) and outer plexiform layer (OPL). Structural gaps, equivalent to "retinoschisis cavities," are evident in the INL. Photoreceptors are reduced in the retinal sections in the $\mathrm{KO}$ mice versus normal mice, and some photoreceptor nuclei localize to the inner and outer segment zones (IS and OS) rather than exclusively within the outer nuclear layer (ONL). As in XLRS humans, the Rs1-KO mouse ERG shows disproportionate b-wave reduction across the range of stimulus flash intensity, as is readily seen in the ERG wave form (arrows) and also in the graph of b-wave amplitude versus intensity. (From Zeng et al. 2004; reprinted, with express permission from the authors.)

et al. 2005; Campochiaro et al. 2006; Bainbridge et al. 2008; Hauswirth et al. 2008; Maguire et al. 2008, 2009; Simonelli et al. 2010; Balaggan and Ali 2012). Today, a large number of gene therapy constructs, in particular those for human ocular diseases, are utilizing AAV. AAV is a nonpathogenic dependovirus that replicates in the presence of another active virus infection, often adenovirus or herpes simplex virus (Berns and Giraud 1996). AAV virions are nonenveloped and typically encase single stranded DNA. There are at least 12 naturally occurring serotypes of AAV (designated AAV1-AAV12), although more than 100 nonredundant forms have been discovered (Mori et al. 2004; Daya and Berns 2008; Vandenberghe and Auricchio 2012).

AAV has two open reading frames. The first contains rep, which encodes four proteins necessary for viral replication. The second comprises cap, which encodes three structural proteins that make up the outer icosahedral capsid of the virus. The cap or capsid proteins have different affinities for various cell surface receptors. For instance, the primary receptors for AAV serotype 2 (AAV2) are heparin sulphate and $\alpha_{V} \beta_{5}$ integrins (Summerford and Samulski 1998; Summerford et al. 1999). AAV4 and AAV5 preferentially bind sialic acid or platelet-derived 
growth factor (Kaludov et al. 2002; Di Pasquale et al. 2003), whereas AAV3, AAV8, and AAV9 bind to laminin (Akache et al. 2006). Therefore, careful selection of viral serotype becomes an important consideration in choosing an appropriate vector for gene delivery for a given disease. This and the delivery route may be critical for a successful gene-based interventional approach.

Choosing the correct viral vector can help address another key component of a successful gene therapy product, namely the appropriate expression of the replacement or restorative gene. Historically, transgene expression from AAV vectors has a delayed onset, sometimes requiring up to 6 wk to achieve peak levels (Bennett et al. 1997). This would be detrimental for acute applications. However, two strategies have been devised to overcome this limitation. The first is the development of modified or mutant capsids. Mutant capsids with specific amino acid substitutions have resulted in higher transgene expression in a shorter period of time (Petrs-Silva et al. 2009). Investigators also have found that photoreceptors can be effectively transduced by intravitreal administration, a finding that has practical clinical implications for both ophthalmologists and patients (see below) (Dalkara et al. 2011; MacLachlan et al. 2011; Petrs-Silva et al. 2011).

In addition to the gene delivery vector, the second consideration is the rate-limiting requirement for synthesis of a complementary DNA strand from the original AAV single-strand DNA. Incorporating a second strand of DNA within the virus (self-complementary AAV) reduces the time required for gene expression by removing the necessity to synthesize a second DNA strand. This also eliminates the need for host DNA replication machinery. Using this approach it has been shown that transgene expression can be measured within $4 \mathrm{~d}$ following $\mathrm{AAV}$-transgene introduction into the eye (Pang et al. 2010).

A third component that is often critical for the pharmacokinetic expression of the transgene is promoter selection. The cytomegalovirus (CMV) promoter is commonly used. This promoter can lead to high transgene expression but in many systems the expression is transient, promiscuous and in all cell types. Because XLRS involves chronic degeneration of retinal cells over years and decades in humans, an important criterion is selection of a promoter that leads to prolonged gene production in multiple cell types. Thus, for XLRS, several promoters including the rhodopsin (rho) and rhodopsin kinase (rho K) promoters have been investigated. Both of these promoters are specific to photoreceptors and give high expression, but they may also result in cell death, likely because of overexpression of retinoschisin (Y Zeng, unpubl.). Use of the retinoschisis promoter should facilitate the expression of retinoschisin in all cells in which it is normally found. By including the interphotoreceptor retinoid binding protein enhancer, gene expression levels following intravitreal injection reaches, in some animals, levels comparable to that found in wild-type animals (see Fig. 4).

\section{Routes of Administration}

In the majority of ocular gene therapy trials using $\mathrm{AAV}$, the genes are delivered via subretinal injection (Bainbridge et al. 2008; Hauswirth et al. 2008; Maguire et al. 2008, 2009; Simonelli et al. 2010) with good success. This route of administration has the experimental advantage of direct dose scaling from the small mouse eye to the larger human eye. However, this procedure is complex and requires pars plana vitrectomy surgery in an operating room, which has a high risk of complications (e.g., retinal tear or retinal detachment). XLRS patients are already prone to retinal detachment, thus utilizing a method that decreases this occurrence would be preferred. Intravitreal injections into the eye have become commonplace with the advent of Macugen, Lucentis, and Eylea (three agents approved for the treatment of wet age-related macular degeneration), and can be repeated monthly. Moreover, intravitreal injections can be administered in the office and given under topical anesthesia, making this procedure less complicated and more convenient for patient, physician, and clinical staff. However, there is 
R.A. Bush et al.
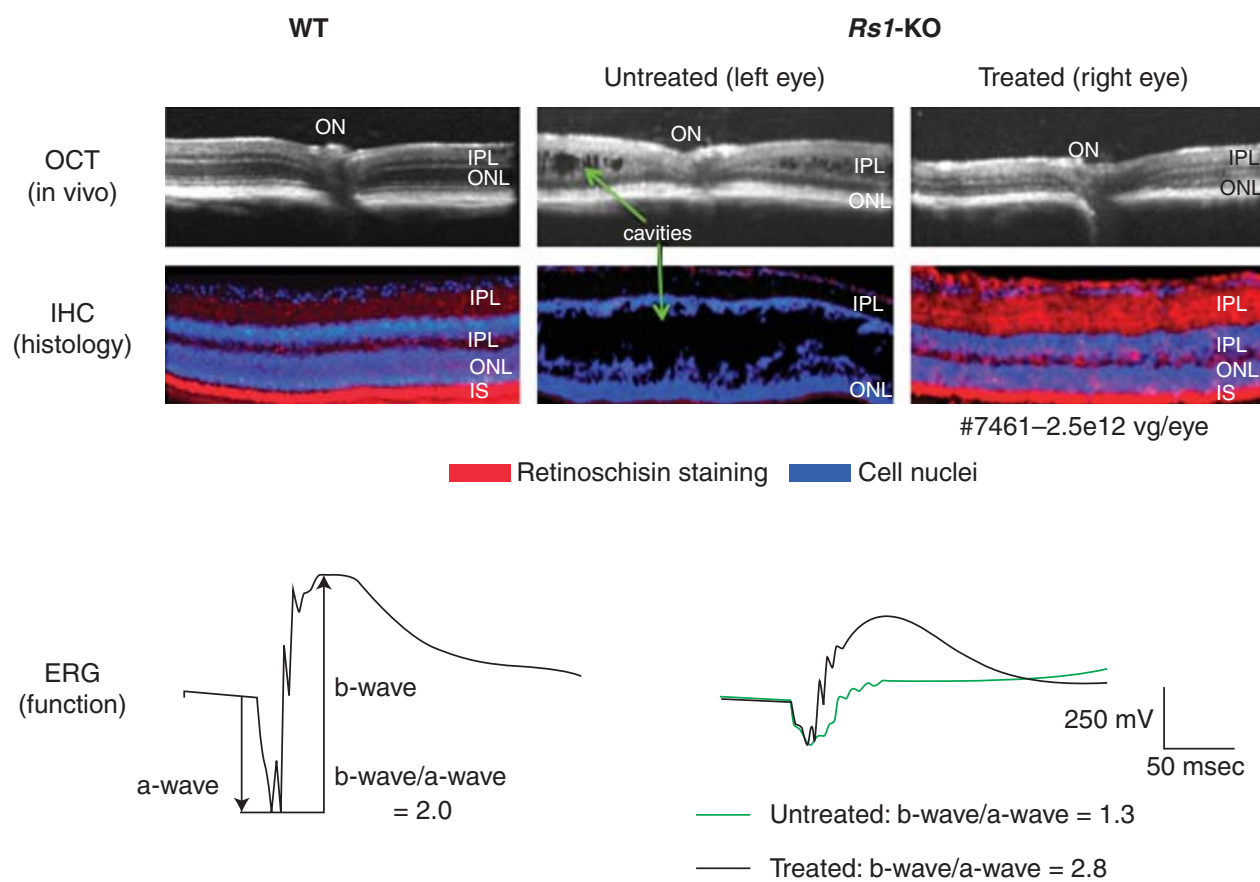

Figure 4. Retinal structure, retinoschisin expression and function in wild-type (WT), untreated- and treatedRs1-KO mice. OCT in vivo images show the structure of a portion of the retinas centered on the optic nerve $(\mathrm{ON})$ from a WTmouse and the right and left retinas of an Rs1-KO mouse treated in the right eye with $2.5 \times 10^{9}$ $\mathrm{vg} /$ eye of AAV8-retinoschisin vector. The darker bands are the nuclear layers of the retina, the thickest being the outer nuclear layer (ONL) containing the photoreceptor cells. The lighter bands above the ONL are the outer and inner plexiform (IPL) layers. In the untreated eye of the Rs1-KO mouse, these layers are much less dense and the borders between the layers less distinct. There are large cavities spanning the region between the ONL and IPL, a region that contains the second order neurons, including bipolar cells and their synapses with photoreceptors. The right eye was treated at day P21 and evaluated 16 wk later. OCT shows the cavities have closed in the treated retina, and the borders between the layers are more regular and distinct than in the untreated eyes. The layer margins are still less distinct than in the WT retina. Immunohistochemical (IHC) staining for retinoschisin protein (red) in the WT retina shows its presence in the plexiform layers, with particularly strong staining in the inner segments (IS) of the photoreceptors. Specific staining for retinoschisin is completely lacking in the untreated eye of the Rs1-KO mouse but is very strong in the treated eye, indicating robust expression of the therapeutic protein in the same cells as in WT. The faint staining below the ONL in the untreated eye is nonspecific staining of the retinal pigment epithelium. Immunohistochemistry analyses show that retinoschisin expression in photoreceptor inner segments in treated eyes are essentially comparable to WT. Image brightness intensity was adjusted against blue nuclear staining of DAPI. The ERG shows a-wave and b-wave responses to a bright flash of light, indicating phototransduction activity of photoreceptors and activity of the second order bipolar cells, respectively. Both are reduced in size in the Rs1-KO mouse retinas, but the ratio of the b-wave to the a-wave is pathologically reduced in the untreated eye while it is increased in the treated retina. This is consistent with the structural findings indicating severe pathology in the retina postsynaptic to photoreceptors in untreated retinas, with structure partially maintained or restored when treated by AAV8retinoschisin. In most of our studies the vector is administered at day P21-P28, when the retina is young but fully mature. However, similar rescue of structure and function is observed when AAV8-retinoschisin is administered at 2 and 3 mo of age. 
not a clear method to scale from a small animal eye to the larger human eye.

Intravitreal administration of native AAV vectors has resulted in scattered and spotty transduction of ganglion cells at the surface of the retina, but the vectors do not penetrate to reach neurons deep in the rodent or monkey eye (Dalkara et al. 2013). AAV2 variants with tyrosine mutations, or newer laboratory-created AAV vectors with capsid modifications, show the ability to penetrate deep into the retina (Petrs-Silva et al. 2009; Vandenberghe and Auricchio 2012; Dalkara et al. 2013). Consequently, intravitreal delivery of genes for retinal degenerative diseases is becoming more promising clinically. In the case of XLRS, data using an AAV8 containing the RS1 gene (AAV8-retinoschisin) delivered into the eyes of XLRS mice by intravitreal injection show robust RS1 staining of neurons throughout the mouse retina, including photoreceptors, bipolar and amacrine cells (Park et al. 2009). This appears specific for the pathophysiology caused by XLRS, as the same vector does not penetrate the wild-type mouse retina. Additionally, a clinical trial utilizing AAV2 with soluble Flt for wet age-related macular degeneration (NCT01024998) is using intravitreal delivery, providing further support that intraocular injection is plausible for some vectors. The published preclinical studies using AAV2 to deliver an antiangiogenic molecule in primate eyes showed intraocular inflammation to the vector capsid, as the AAV2-null vector gave similar findings (MacLachlan et al. 2011). Inflammation was transitory, initially appearing in some animals at 1 mo after intravitreal injection, increasing over the next few months, and then declining.

\section{Transition to Efficacy Studies}

Preclinical safety and efficacy studies in animals can provide the foundation for human clinical trials and are helpful to establish a possible benefit to treatment that outweighs potential risks. As reviewed above, mice in which expression of the Rs1 gene has been disrupted resulting in retinoschisin knockout ( $R s 1-\mathrm{KO})$ mice are the only animal models for XLRS. Structural and functional studies of Rs1-KO mice have shown a phenotype that closely mimics retinal pathology in XLRS (Zeng et al. 2004). Cavity formation reaches a maximum by $4-6$ mo, in parallel with functional changes such as reduction in the ERG b-wave to a-wave ratio (b/a ratio) (Kjellstrom et al. 2007). This change is comparable to XLRS in young adults when the ERG $\mathrm{b}$-wave and $\mathrm{b} / \mathrm{a}$ ratio tends to reach minimum in the most severe patients (Bowles et al. 2011). AAV8-retinoschisin efficacy studies in our laboratory using Rs1-KO mice are described below. Retinal function was measured using ERGs, and retinal structure was evaluated in vivo with OCT imaging. Both are common clinical measures and are performed using identical or similar instruments to those used for human clinical diagnosis. In addition, retinoschisin expression was evaluated postmortem in treated animals using semiquantitative immunohistochemistry. This provides a combined assessment of therapeutic protein expression with both structural and functional benefit following vector administration.

\section{Efficacy Study Results}

Two groups of Rs1-KO mice received intravitreal injections in one eye between 3 and 5 wk of age of either an AAV8 vector carrying a self-complimentary copy of the human retinoschisin gene and promoter or vehicle alone. Injected doses were $2.5 \times 10^{9}$ vector genomes $(\mathrm{vg}) /$ eye down to $1 \times 10^{6} \mathrm{vg} /$ eye. ERGs were recorded between 12 and 16 wk after injection, followed within 2 wk by OCT imaging and removal of the eyes for immunohistochemistry to evaluate retinoschisin expression. Figure 4 shows the results from the treated and untreated eyes of a single mouse injected with $2.5 \times 10^{9} \mathrm{vg} /$ eye and evaluated $16 \mathrm{wk}$ after injection compared with a wild-type mouse. The untreated eye of the Rs1-KO mouse has large cavities spanning the layers between the photoreceptor outer nuclear layer and the inner plexiform layer. These intermediate layers are also thicker than in wild type and have ill-defined boundaries, indicating a degree of disorganization compared with the well-defined layers in the wild-type animal. 
R.A. Bush et al.

The cavities in the untreated retina appear as empty space in the histological sections, but in vivo there are structural connections between the inner and outer margins of the cavities similar to those in human XLRS (Fig. 1). The untreated eye lacks retinoschisin expression, which is apparent in the inner nuclear layer and outer plexiform layer and is strongest in the inner segments of the photoreceptors in the wildtype retina. The structure of the treated retina in vivo and in histological sections, however, is similar to wild type in organization of the retinal layers and lack of cavities. Some disorganization is still evident in the internal structure of the layers and the indistinct or irregular margins of the layers. The treated retina shows heavy retinoschisin staining of inner segments and in the two plexiform layers between the rows of retinal cell bodies. Retinoschisin expression in this retina equals or exceeds that of wild type, although this is variable across injected eyes and sometimes within injected eyes.

The ERG of the wild-type mouse shows a dark-adapted a-wave and b-wave in response to a bright stimulus flash. The a-wave reflects phototransduction in the rods, and the b-wave originates from bipolar cells postsynaptic to the photoreceptors. Thus, a reduction of photore- ceptor number or activity will produce a reduction in both the a-wave and b-wave, because postsynaptic responses depend on photoreceptor input. A reduction in the a-wave greater than in the b-wave, that is, an increase in the $b / a$ ratio, is a hallmark of photoreceptor degenerations (Machida et al. 2000), because photoreceptors greatly outnumber the bipolar cells, and this convergence tends to reduce the effect of photoreceptor loss vis-à-vis the b-wave generation. The response from the untreated eye of the Rs1-KO mouse in Figure 4 shows a reduction in $\mathrm{b} / \mathrm{a}$ ratio, indicating a disproportionate effect on the response of bipolar cells. This could be a result of structural and functional disruption of bipolar cells themselves and/or the synaptic signaling between photoreceptors and these second order neurons. The smaller a-wave compared with wild type indicates that there was substantial photoreceptor loss, which is also apparent as a reduction in the outer nuclear layer, as shown by OCT and histology. The increased $\mathrm{b} / \mathrm{a}$ wave ratio in treated eyes is consistent with the relatively normal structural appearance of the retinal layers postsynaptic to the photoreceptors, coupled with a substantial loss of photoreceptors. Figure 5 shows dose-response results for Rs1-KO eyes injected with AAV8-ret-
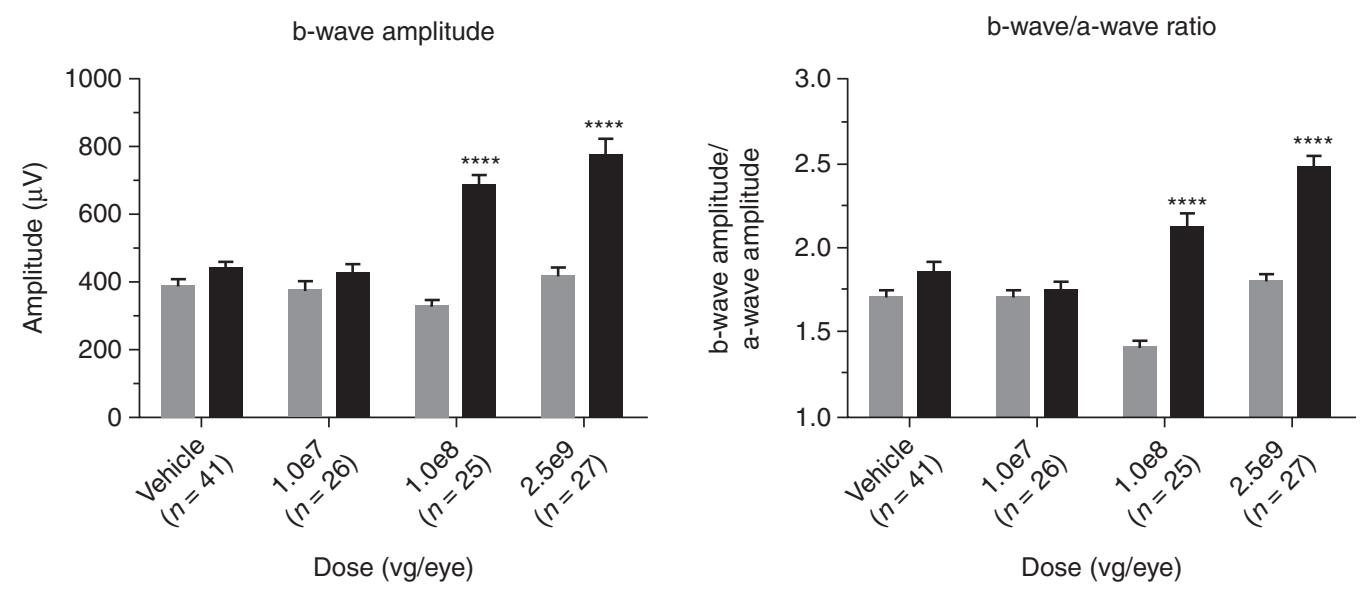

Figure 5. Dose response graphs for the ERG b-wave and b-wave to a-wave ratio in Rs1-KO mice treated with AAV8-retinoschisin. Rs1-KO mice were treated in one eye with different concentrations of vector or vehicle between 3 and $5 \mathrm{wk}$ of age, and the ERG was recorded 12 to $16 \mathrm{wk}$ after injection. The b-wave and the b-wave to a-wave ratio was significantly increased at doses of $1 \mathrm{e} 8$ and $2.5 \mathrm{e} 9$, relative to untreated eyes (multiple $t$-tests using Holm-Sidak correction for multiple comparisons). 
inoschisin or vector controls. There was a significant improvement in the $\mathrm{b}$-wave and $\mathrm{b} / \mathrm{a}$ wave ratio in eyes treated with $1 \times 10^{8}$ and 2.5 $\times 10^{9} \mathrm{vg} /$ eye of AAV8-retinoschisin but not with $1 \times 10^{7} \mathrm{vg} /$ eye or vehicle. The size and number of cavities were significantly reduced at $1 \times 10^{8}$ and $2.5 \times 10^{9} \mathrm{vg} /$ eye, and these retinas showed significant retinoschisin protein expression, as assessed with immunohistochemistry, compared with untreated eyes (Fig. 4)

\section{CONCLUDING REMARKS}

Based on the very promising mouse efficacy studies, the strong transduction of the retina using AAV8 after intravitreous administration, and an acceptable safety profile in the Rs1-KO mouse, a next step is to advance to a phase I clinical trial in XLRS patients. The data obtained to date from animal studies suggest several issues to be considered in designing early clinical trials of $R S 1$ gene therapy. Each patient should be followed carefully for any clinical indication of an ocular inflammatory response to vector administration. Simultaneously, therapeutic benefit should also be measured. The first subjects for human treatment studies may have more severe disease, in which vision is already substantially compromised. Each patient should be carefully monitored to ensure that there is no detrimental effect. Then individuals with more moderate disease might be considered for treatment. In designing a human study, clinical examination methods that identify underlying cellular dysfunction should be used, if possible. In a Phase I safety study, these might include reading evaluation to monitor visual acuity, degree of ERG impairment, and OCT imaging to monitor the structure of the retina. The principles learned from genetic analyses in XLRS patients and efficacy studies in XLRS mice support clinical testing of a RS1 gene therapy approach to reduce vision loss in retinoschisis patients.

\section{ACKNOWLEDGMENTS}

The authors are grateful to Dr. Catherine Cukras for the fundus photo and OCT scan from an
XLRS patient and Cindy Best, Matthew McMahon, and Santa Tumminia for their assistance in the preparation of this review. Supported by the National Institutes of Health (NIH) Intramural Research Program through the National Institute on Deafness and Other Communication Disorders and the National Eye Institute.

\section{REFERENCES}

Akache B, Grimm D, Pandey K, Yant SR, Xu H, Kay MA. 2006. The 37/67-kilodalton laminin receptor is a receptor for adeno-associated virus serotypes 8, 2, 3, and 9 J Virol 80: 9831-9836.

Apushkin MA, Fishman GA. 2006. Use of dorzolamide for patients with X-linked retinoschisis. Retina 26: $741-745$.

Bainbridge JW, Smith AJ, Barker SS, Robbie S, Henderson R, Balaggan K, Viswanathan A, Holder GE, Stockman A Tyler N, et al. 2008. Effect of gene therapy on visual function in Leber's congenital amaurosis. N Engl J Med 358: 2231-2239.

Balaggan KS, Ali RR. 2012. Ocular gene delivery using lentiviral vectors. Gene Ther 19: 145-153.

Bennett J, Duan D, Engelhardt JF, Maguire AM. 1997. Realtime, noninvasive in vivo assessment of adeno-associated virus-mediated retinal transduction. Invest Ophthalmol Vis Sci 38: 2857-2863.

Berns KI, Giraud C. 1996. Biology of adeno-associated virus. Curr Top Microbiol Immunol 218: 1-23.

Bowles K, Cukras C, Turriff A, Sergeev Y, Vitale S, Bush RA, Sieving PA. 2011. X-linked retinoschisis: RS1 mutation severity and age affect the ERG phenotype in a cohort of 68 affected male subjects. Invest Ophthalmol Vis Sci 52: 9250-9256.

Campochiaro PA, Nguyen QD, Shah SM, Klein ML, Holz E, Frank RN, Saperstein DA, Gupta A, Stout JT, Macko J, et al. 2006. Adenoviral vector-delivered pigment epithelium-derived factor for neovascular age-related macular degeneration: Results of a phase I clinical trial. Hum Gene Ther 17: 167-176.

Chevez-Barrios P, Chintagumpala M, Mieler W, Paysse E, Boniuk M, Kozinetz C, Hurwitz MY, Hurwitz RL. 2005. Response of retinoblastoma with vitreous tumor seeding to adenovirus-mediated delivery of thymidine kinase followed by ganciclovir. J Clin Oncol 23: 7927-7935.

Dalkara D, Kolstad KD, Guerin KI, Hoffmann NV, Visel M, Klimczak RR, Schaffer DV, Flannery JG. 2011. AAV mediated GDNF secretion from retinal glia slows down retinal degeneration in a rat model of retinitis pigmentosa. Mol Ther 19: 1602-1608.

Dalkara D, Byrne LC, Klimczak RR, Visel M, Yin L, Merigan WH, Flannery JG, Schaffer DV. 2013. In vivo-directed evolution of a new adeno-associated virus for therapeutic outer retinal gene delivery from the vitreous. Sci Transl Med 5: 189ra176.

Daya S, Berns KI. 2008. Gene therapy using adeno-associated virus vectors. Clin Microbiol Rev 21: 583-593. 
R.A. Bush et al.

Di Pasquale G, Davidson BL, Stein CS, Martins I, Scudiero D, Monks A, Chiorini JA. 2003. Identification of PDGFR as a receptor for AAV-5 transduction. Nat Med 9: $1306-$ 1312.

Eksandh LC, Ponjavic V, Ayyagari R, Bingham EL, Hiriyanna KT, Andreasson S, Ehinger B, Sieving PA. 2000. Phenotypic expression of juvenile $\mathrm{X}$-linked retinoschisis in Swedish families with different mutations in the XLRS1 gene. Arch Ophthalmol 118: 1098-1104.

George ND, Yates JR, Moore AT. 1995. X linked retinoschisis. Br J Ophthalmol 79: 697-702.

Ghajarnia M, Gorin MB. 2007. Acetazolamide in the treatment of X-linked retinoschisis maculopathy. Arch Ophthalmol 125: 571-573.

Grayson C, Reid SN, Ellis JA, Rutherford A, Sowden JC, Yates JR, Farber DB, Trump D. 2000. Retinoschisin, the $\mathrm{X}$-linked retinoschisis protein, is a secreted photoreceptor protein, and is expressed and released by Weri-Rb1 cells. Hum Mol Genet 9: 1873-1879.

Haas J. 1898. Ueber das zusammenvorkommen von veraenderungen der retina und choroidea. Arch Augenheilkd 37: 343-348.

Hauswirth W, Aleman TS, Kaushal S, Cideciyan AV, Schwartz SB, Wang L, Conlon T, Boye SL, Flotte TR, Byrne B, et al. 2008. Phase I trial of Leber congenital amaurosis due to RPE65 mutations by ocular subretinal injection of adeno-associated virus gene vector: Shortterm results. Hum Gene Ther 0: 081015093227032.

Ikeda F, Iida T, Kishi S. 2008. Resolution of retinoschisis after vitreous surgery in X-linked retinoschisis. Ophthalmology 115: 718-722 e711.

Jablonski MM, Dalke C, Wang X, Lu L, Manly KF, Pretsch W, Favor J, Pardue MT, Rinchik EM, Williams RW, et al. 2005. An ENU-induced mutation in Rs $1 h$ causes disruption of retinal structure and function. Mol Vis 11: 569581.

Kaludov N, Handelman B, Chiorini JA. 2002. Scalable purification of adeno-associated virus type 2, 4, or 5 using ion-exchange chromatography. Hum Gene Ther 13: $1235-1243$.

Kaplan J, Pelet A, Hentati H, Jeanpierre M, Briard ML, Journel H, Munnich A, Dufier JL. 1991. Contribution to carrier detection and genetic counselling in $\mathrm{X}$ linked retinoschisis. J Med Genet 28: 83-388.

Kjellstrom S, Bush RA, Zeng Y, Takada Y, Sieving PA. 2007. Retinoschisin gene therapy and natural history in the $R s 1 h$-KO mouse: Long-term rescue from retinal degeneration. Invest Ophthalmol Vis Sci 48: 3837-3845.

Machida S, Kondo M, Jamison JA, Khan NW, Kononen LT, Sugawara T, Bush RA, Sieving PA. 2000. P23H rhodopsin transgenic rat: Correlation of retinal function with histopathology. Invest Ophthalmol Vis Sci 41: 3200-3209.

MacLachlan TK, Lukason M, Collins M, Munger R, Isenberger E, Rogers C, Malatos S, Dufresne E, Morris J, Calcedo R, et al. 2011. Preclinical safety evaluation of AAV2-sFLT01-A gene therapy for age-related macular degeneration. Mol Ther 19: 326-334.

Maguire AM, Simonelli F, Pierce EA, Pugh EN Jr, Mingozzi F, Bennicelli J, Banfi S, Marshall KA, Testa F, Surace EM, et al. 2008. Safety and efficacy of gene transfer for Leber's congenital amaurosis. N Engl J Med 358: 2240-2248.
Maguire AM, High KA, Auricchio A, Wright JF, Pierce EA, Testa F, Mingozzi F, Bennicelli JL, Ying GS, Rossi S, et al. 2009. Age-dependent effects of RPE65 gene therapy for Leber's congenital amaurosis: A phase 1 dose-escalation trial. Lancet 374: 1597-1605.

Mann I, MacRae A. 1938. Congenital vascular veils in the vitreous. Br J Ophthalmol 22: 1-10.

Min SH, Molday LL, Seeliger MW, Dinculescu A, Timmers AM, Janssen A, Tonagel F, Tanimoto N, Weber BH, Molday RS, et al. 2005. Prolonged recovery of retinal structure/function after gene therapy in an Rsih-deficient mouse model of X-linked juvenile retinoschisis. Mol Ther 12: 644-651.

Molday LL, Hicks D, Sauer CG, Weber BH, Molday RS. 2001. Expression of X-linked retinoschisis protein RS1 in photoreceptor and bipolar cells. Invest Ophthalmol Vis Sci 42: 816-825.

Molday RS, Kellner U, Weber BH. 2012. X-linked juvenile retinoschisis: Clinical diagnosis, genetic analysis, and molecular mechanisms. Prog Retin Eye Res 31: 195-212.

Mori S, Wang L, Takeuchi T, Kanda T. 2004. Two novel adeno-associated viruses from cynomolgus monkey: Pseudotyping characterization of capsid protein. Virology 330: 375-383.

Pang J, Boye SE, Lei B, Boye SL, Everhart D, Ryals R, Umino Y, Rohrer B, Alexander J, Li J, et al. 2010. Self-complementary AAV-mediated gene therapy restores cone function and prevents cone degeneration in two models of Rpe65 deficiency. Gene Ther 17: 815-826.

Park TK, Wu Z, Kjellstrom S, Zeng Y, Bush RA, Sieving PA, Colosi P. 2009. Intravitreal delivery of AAV8 retinoschisin results in cell type-specific gene expression and retinal rescue in the Rs1-KO mouse. Gene Ther 16: 916-926.

Peachey N, Fishman G, Derlacki D, Brigell M. 1987. Psychophysical and electroretinographic findings in X-linked juvenile retinoschisis. Arch Ophthalmol 105: 513-516.

Petrs-Silva H, Dinculescu A, Li Q, Min SH, Chiodo V, Pang JJ, Zhong L, Zolotukhin S, Srivastava A, Lewin AS, et al. 2009. High-efficiency transduction of the mouse retina by tyrosine-mutant AAV serotype vectors. Mol Ther 17: 463-471.

Petrs-Silva H, Dinculescu A, Li Q, Deng WT, Pang JJ, Min SH, Chiodo V, Neeley AW, Govindasamy L, Bennett A, et al. 2011. Novel properties of tyrosine-mutant AAV2 vectors in the mouse retina. Mol Ther 19: 293-301.

Prenner JL, Copone A Jr, Ciaccia S, Takada Y, Sieving PA, Trese MT. 2006. Congenetital X-linked retinoschisis classification system. Retina 26: S61-S64.

Reid SN, Akhmedov NB, Piriev NI, Kozak CA, Danciger M, Farber DB. 1999. The mouse X-linked juvenile retinoschisis cDNA: Expression in photoreceptors. Gene 227: 257-266.

Reid SN, Yamashita C, Farber DB. 2003. Retinoschisin, a photoreceptor-secreted protein, and its interaction with bipolar and muller cells. J Neurosci 23: 6030-6040.

Sauer CG, Gehrig A, Warneke-Wittstock R, Marquardt A, Ewing CC, Gibson A, Lorenz B, Jurklies B, Weber BH. 1997. Positional cloning of the gene associated with $X$ linked juvenile retinoschisis. Nat Genet 17: 164-170.

Sergeev YV, Caruso RC, Meltzer MR, Smaoui N, MacDonald IM, Sieving PA. 2010. Molecular modeling of retino- 
schisin with functional analysis of pathogenic mutations from human X-linked retinoschisis. Hum Mol Genet 19: 1302-1313.

Sergeev YV, Vitale S, Sieving PA, Vincent A, Robson AG, Moore AT, Webster AR, Holder GE. 2013. Molecular modeling indicates distinct classes of missense variants with mild and severe XLRS phenotypes. Hum Mol Genet 22: 4756-4767.

Sieving PA, Yashar BM, Ayyagari R. 2000. Juvenile retinoschisis: A model for diagnostic testing of X-linked ophthalmic disease. Trans Am ophthalmol soc 1999;v97: 451-464. Am J Ophthalmol 129: 833.

Simonelli F, Cennamo G, Ziviello C, Testa F, de Crecchio G, Nesti A, Manitto MP, Ciccodicola A, Banfi S, Brancato R, et al. 2003. Clinical features of $\mathrm{X}$ linked juvenile retinoschisis associated with new mutations in the XLRS1 gene in Italian families. Br J Ophthalmol 87: 1130-1134.

Simonelli F, Maguire AM, Testa F, Pierce EA, Mingozzi F, Bennicelli JL, Rossi S, Marshall K, Banfi S, Surace EM, et al. 2010. Gene therapy for Leber's congenital amaurosis is safe and effective through 1.5 years after vector administration. Mol Ther 18: 643-650.

Song JC, McDonnell PJ, Gordon EM, Hall FL, Anderson WF. 2003. Phase I/II evaluation of safety and efficacy of a matrix-targeted retroviral vector bearing a dominant negative cyclin G1 construct (Md-dnG1) as adjunctive intervention for superficial corneal opacity/corneal scarring. Hum Gene Ther 14: 306-309.

Summerford C, Samulski RJ. 1998. Membrane-associated heparan sulfate proteoglycan is a receptor for adeno-associated virus type 2 virions. J Virol 72: 1438-1445.

Summerford C, Bartlett JS, Samulski RJ. 1999. AlphaVbeta5 integrin: A co-receptor for adeno-associated virus type 2 infection. Nat Med 5: 78-82.

Takada Y, Fariss RN, Tanikawa A, Zeng Y, Carper D, Bush R, Sieving PA. 2004. A retinal neuronal developmental wave of retinoschisin expression begins in ganglion cells during layer formation. Invest Ophthalmol Vis Sci 45: 33023312.

The Retinal Consortium. 1998. Functional implications of the spectrum of mutations found in 234 cases with X-linked juvenile retinoschisis. The Retinoschisis Consortium. Hum Mol Genet 7: 1185-1192.
Vandenberghe LH, Auricchio A. 2012. Novel adeno-associated viral vectors for retinal gene therapy. Gene Ther 19: $162-168$.

Vijayasarathy C, Gawinowicz MA, Zeng Y, Takada Y, Bush RA, Sieving PA. 2006. Identification and characterization of two mature isoforms of retinoschisin in murine retina. Biochem Biophys Res Commun 349: 99-105.

Vijayasarathy C, Takada Y, Zeng Y, Bush RA, Sieving PA. 2007. Retinoschisin is a peripheral membrane protein with affinity for anionic phospholipids and affected by divalent cations. Invest Ophthalmol Vis Sci 48: $991-$ 1000.

Vincent A, Robson AG, Neveu MM, Wright GA, Moore AT, Webster AR, Holder GE. 2013. A phenotype-genotype correlation study of X-linked retinoschisis. Ophthalmology 120: 1454-1464.

Vogel WF, Abdulhussein R, Ford CE. 2006. Sensing extracellular matrix: An update on discoidin domain receptor function. Cell Signal 18: 1108-1116.

Weber BH, Schrewe H, Molday LL, Gehrig A, White KL, Seeliger MW, Jaissle GB, Friedburg C, Tamm E, Molday RS. 2002. Inactivation of the murine $\mathrm{X}$-linked juvenile retinoschisis gene, $R s 1 h$, suggests a role of retinoschisin in retinal cell layer organization and synaptic structure. Proc Natl Acad Sci 99: 6222-6227.

Wilczek M. 1935. Ein der netzhautspaltung (retinoschisis) mit einer offnung. Zeit Augenhlkd 85: 105-116.

Wu WW, Molday RS. 2003. Defective discoidin domain structure, subunit assembly, and endoplasmic reticulum processing of retinoschisin are primary mechanisms responsible for X-linked retinoschisis. J Biol Chem 278: 28139-28146.

Zeng Y, Takada Y, Kjellstrom S, Hiriyanna K, Tanikawa A, Wawrousek E, Smaoui N, Caruso R, Bush RA, Sieving PA. 2004. RS-1 gene delivery to an adult Rsih knockout mouse model restores ERG b-wave with reversal of the electronegative waveform of $\mathrm{X}$-linked retinoschisis. Invest Ophthalmol Vis Sci 45: 3279-3285.

Zhour A, Bolz S, Grimm C, Willmann G, Schatz A, Weber BH, Zrenner E, Fischer MD. 2012. In vivo imaging reveals novel aspects of retinal disease progression in $R s 1 h^{-/ Y}$ mice but no therapeutic effect of carbonic anhydrase inhibition. Vet Ophthalmol 15: 123-133. 


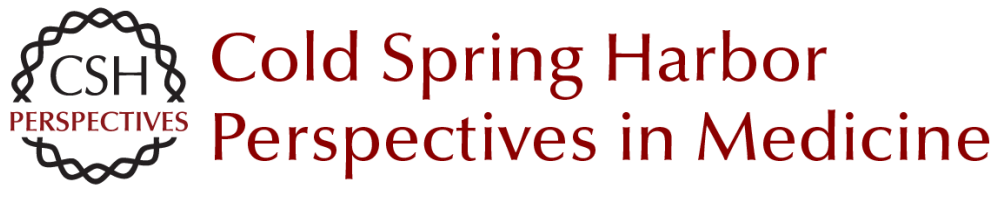

\title{
Convergence of Human Genetics and Animal Studies: Gene Therapy for X-Linked Retinoschisis
}

\author{
Ronald A. Bush, Lisa L. Wei and Paul A. Sieving
}

Cold Spring Harb Perspect Med 2015; doi: 10.1101/cshperspect.a017368 originally published online June 22, 2015

Subject Collection Retinal Disorders: Genetic Approaches to Diagnosis and Treatment

Trial by "Firsts": Clinical Trial Design and

Regulatory Considerations in the Development

and Approval of the First AAV Gene Therapy

Product in the United States

Kathleen Z. Reape and Katherine A. High

Immunology of Retinitis Pigmentosa and Gene

Therapy-Associated Uveitis

Paul Yang, Debarshi Mustafi and Kathryn L. Pepple

Developing New Vectors for Retinal Gene Therapy

Emilia A. Zin, Bilge E. Ozturk, Deniz Dalkara, et al.

Beyond the NEI-VFQ: Recent Experience in the

Development and Utilization of Patient-Reported

Outcomes for Inherited Retinal Diseases

Todd Durham, Judit Banhazi, Francesco Patalano, et al.

Electronic Retinal Prostheses

Daniel Palanker

Alternative RNA Splicing in the Retina: Insights and Perspectives

Casey J. Keuthan, Sadik Karma and Donald J. Zack

X-Linked Retinoschisis

Cristy A. Ku, Lisa W. Wei and Paul A. Sieving

A Systematic Review of Optogenetic Vision Restoration: History, Challenges, and New Inventions from Bench to Bedside

Antonia Stefanov and John G. Flannery
Lessons Learned from the Development of the

First FDA-Approved Gene Therapy Drug,

Voretigene Neparvovec-rzyl

Jean Bennett and Albert M. Maguire

Therapeutic Gene Editing in Inherited Retinal Disorders Jinjie Ling, Laura A. Jenny, Ashley Zhou, et al.

Cell-Based Therapies: Strategies for Regeneration Marina Pavlou and Thomas A. Reh

The Importance of Natural History Studies in Inherited Retinal Diseases Allison Ayala, Janet Cheetham, Todd Durham, et al.

\section{Photoreceptor Cell Replacement Using \\ Pluripotent Stem Cells: Current Knowledge and \\ Remaining Questions}

Christelle Monville, Olivier Goureau and Karim Ben M'Barek

iPSC-RPE in Retinal Degeneration: Recent

Advancements and Future Perspectives

Tadao Maeda and Masayo Takahashi

Retinal Degeneration Animal Models in Bardet-

Biedl Syndrome and Related Ciliopathies

Clarisse Delvallée and Hélène Dollfus

Mobility Testing and Other Performance-Based

Assessments of Functional Vision in Patients with Inherited Retinal Disease

Daniel Chung, Colas Authié and Laure Blouin

For additional articles in this collection, see http://perspectivesinmedicine.cshlp.org/cgi/collection/ 
For additional articles in this collection, see http://perspectivesinmedicine.cshlp.org/cgi/collection/ 